\title{
Giant Hernia with Incarceration
}

\author{
Amadou Maïga1*, Amadou Bah"1, Ibrahim Diakité1, Zakari Saye1, Boubacar Y. Sidibé1, \\ Bathio Traoré2, Moussa Diassana ${ }^{2}$, Siaka Konaté ${ }^{1}$, Arouna A. Doumdia ${ }^{1}$, Tani Koné1, \\ Aly B. Diallo², Amadou A. Traoré1, Mamadou Diallo³, Bakary T. Dembélé1, \\ Oumou H. Saadé1, Moussa Kantée, Madiassa Konaté1, Souleymane Dembélé1, \\ Moussa Samaké1, Moussa Konaté3, Lassana Kanté1, Alhassane Traoré1
}

${ }^{1}$ Service de chirurgie générale, hôpital universitaire, Bamako, Mali

${ }^{2}$ Service de chirurgie générale, hôpital de Sikasso, Sikasso, Mali

${ }^{3}$ Centre de référence de la Commune VI, Bamako, Mali

Email: *amadoumaiga3@gmail.com

How to cite this paper: Maïga, A., Bah, A., Diakité, I., Saye, Z., Sidibé, B.Y., Traoré, B., Diassana, M., Konaté, S., Doumdia, A.A., Koné, T., Diallo, A.B., Traoré, A.A., Diallo, M., Dembélé, B.T., Saadé, O.H., Kanté, M., Konaté, M., Dembélé, S., Samaké, M., Konaté, M., Kanté, L. and Traoré, A. (2021) Giant Hernia with Incarceration. Surgical Science, 12, 399-403.

https://doi.org/10.4236/ss.2021.1212042

Received: March 24, 2021

Accepted: December 25, 2021

Published: December 28, 2021

Copyright $\odot 2021$ by author(s) and Scientific Research Publishing Inc. This work is licensed under the Creative Commons Attribution International License (CC BY 4.0).

http://creativecommons.org/licenses/by/4.0/ (c) (i) Open Access

\begin{abstract}
Introduction: Giant hernias induce changes which reduce the quality of life of patients and make their surgical management complex. Adequate preoperative preparation of the patient guarantees good postoperative progress. It is necessary to avoid resorting to a technique of separation of the compartments during the cure. Here we report the case of a patient who benefited a successful cure using the Ramirez technique. Observation: We report the case of a 60-year-old patient admitted to an outpatient clinic for abdominal swelling evolving for 30 years without the notion of trauma gradually increasing in volume. The interrogation and physical examination led to the diagnosis of a giant white line hernia with incarceration. A preoperative assessment and a preanesthetic consultation were carried out. Intraoperatively, the cecum, transverse colon, sigmoid, jejunum and greater omentum were incarcerated in the bag. After adhesiolysis we proceeded to resect the bag and cure it using the Ramirez technique. The consequences were simple and the patient was discharged on day 4 after her dressing and was seen on day 15, 1 month, 3 months and 1 year. The patient benefited from the placement of an abdominal compression sheath for 3 months. Conclusion: Success in the management of prosthetic material in the absence of prosthetic material depends on good preoperative preparation and the mastery of certain surgical techniques.
\end{abstract}

\section{Keywords}

Giant Hernia, General Surgery, CHU Gabriel Touré

\section{Introduction}

Giant hernias induce physiological changes which reduce the quality of life of 
patients and make their surgical management complex. Adequate preoperative preparation of the patient guarantees good postoperative progress. It is necessary to avoid resorting to a technique of separation of the compartments during the cure. A giant hernia is defined by a collar width greater than $10 \mathrm{~cm}$ [1]. A giant hernia corresponds to a hernia where the hernial content cannot be reduced in the abdominal cavity due to an acute inflammatory reaction inducing congestion of the hernia or adhesions in the hernial sac [2]. If not operated on, these giant hernias can be complicated by strangulation, bowel obstruction, or enterocutaneous fistulization. In practice, all patients with giant hernias must be assessed and then optimized. Optimization includes quitting smoking at least four weeks before the operation, strict glycemic control in diabetics, weight loss, correction of any tissue hypoxemia and nutritional optimization [3]. The closing of the fascial plane has as limiting factors the tension on the fascias and the resulting intra-abdominal hyperpressure. There are several surgical procedures which make up for that. In 1984, Trivellini et al. [4] described the use of discharge incisions on the external oblique muscle in order to decrease tension. In 1990 Ramirez described a technique which will bear his name: the components separation or separation of compartments. It consists of the incision of the tendon of the external oblique beyond the line semi-lunar [5] and the incision of the posterior sheath of the right muscles.

These maneuvers allow to gain in length and ensure medialization of the right muscles. The purpose of this work is to report an observation of a patient operated for giant hernia of the white line with incarceration by the technique of Ramirez with success.

\section{Observation}

We report the case of a 60 -year-old patient admitted to an outpatient clinic for abdominal swelling evolving for 30 years without the notion of trauma gradually increasing in volume. 2 years ago this swelling became painful with no notion of vomiting, but a notion of constipation (4 days without stool) having led the patient to undertake a series of traditional treatment based on oral decoction and fumigation without success, resulting in skin ulceration. Faced with the persistence of pain, she consults us for better care (Figure 1). On admission she had a good general condition (OMS1), pink conjunctiva, no folds of dehydration or malnutrition, blood pressure at 102/70 $\mathrm{mmhg}$, a pulse rate at $84 \mathrm{pulses} / \mathrm{min}$, a temperature at $37.2^{\circ} \mathrm{C}$, a respiratory rate at $18 \mathrm{cycles} / \mathrm{min}$, a weight at $70 \mathrm{~kg}$, a height at $1.58 \mathrm{~m}$, a BMI at 28.04. The patient did not receive a CT scan or MRI due to lack of financial means. An over-umbilical arch with irregular contours of $20 / 15 \mathrm{~cm}$ of long axis (Figure 2), irreducible, the collar was difficult to appreciate on palpation. The pelvic feel was without particularity. The biological assessment carried out was without particularity, so a preanesthetic consultation was requested with an ok for general anesthesia. In the block we made a biconvex lens incision removing skin ulcers; after dissection the collar (Figure 3) was $11 / 12 \mathrm{~cm}$ wide, the contents of the sac were the cecum, the transverse colon, the 


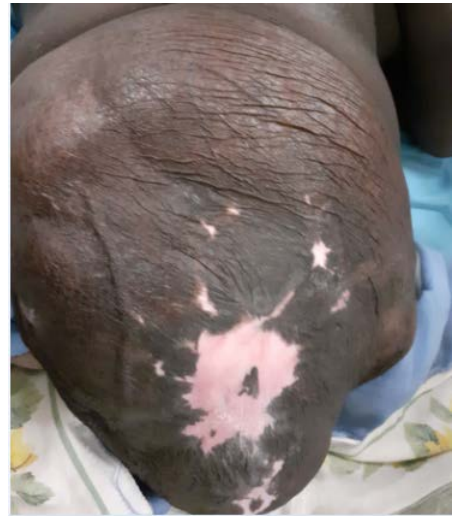

Figure 1. Skin ulceration.

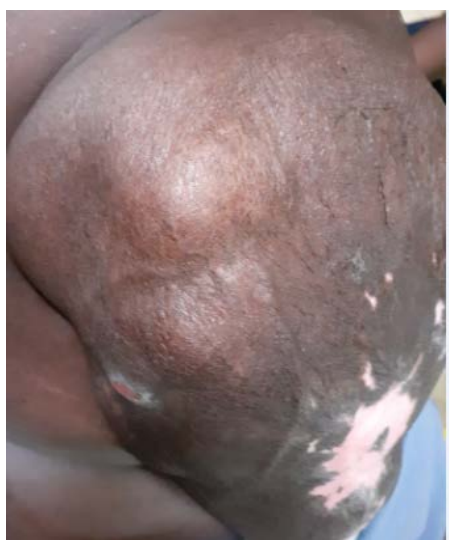

Figure 2. Irregular contours.

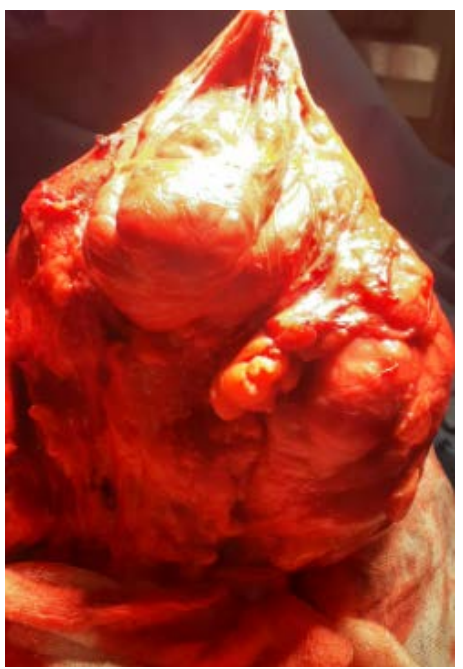

Figure 3. Hernia collar.

sigmoid, the jejunum and the greater omentum (Figure 4). After adhesiolysis we proceeded to resect the bag and cure it using the Ramirez technique. The patient was discharged from the hospital on day 4 after her dressing, with the placement of an abdominal compression sheath for the 3 months. The patient was followed for a year without any particular problem. 


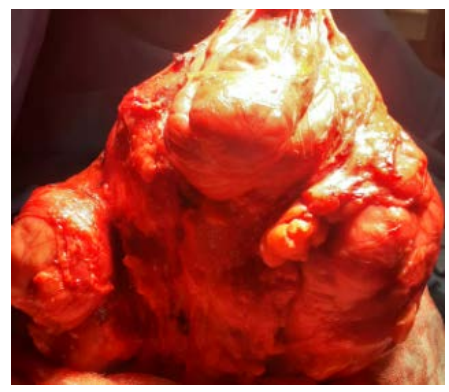

Figure 4. The bag with its contents.

\section{Discussion}

The closure of the fascial plane has as limiting factors the tension on the fascias and the resulting intra-abdominal hyperpressure. There are several surgical procedures that can overcome this. In 1984, Trivellini et al. [4] described the use of discharge incisions on the external oblique muscle in order to decrease tension. In 1990 Ramirez described a technique which will bear his name: the components separation or separation of compartments. It consists of the incision of the tendon of the external oblique beyond the line semi-lunar [5] and the incision of the posterior sheath of the right muscles. These maneuvers allow to gain in length and ensure medialization of the right muscles. There are several abdominal augmentation strategies that aim to increase abdominal volume before proceeding reintegration of hernial content. Among the techniques described, we find progressive preoperative pneumoperitoneum (PPP), the installation of expander type devices and, more recently, the application of botulinum toxin (see below) [6] According to a systematic review of the literature, [6] lePPP allows a net coverage rate of $84 \%$ compared to $92.9 \%$ for expanders and $100 \%$ for botulinum toxin. Note, however, that there were only fourteen patients in the expander category and 29 in the botulinum toxin category versus 229 for PPP. These data are therefore difficult to interpret given the size of the studies, but also their heterogeneity, their level of evidence (absence of randomized study) and a likely selection bias, especially for new techniques. This during abdominal augmentation strategies is not necessarily necessary in large hernias.

\section{Conclusion}

The success in the management in the absence of prosthetic material depends on good preoperative preparation and the mastery of certain surgical techniques.

\section{Conflicts of Interest}

The authors declare no conflicts of interest regarding the publication of this paper.

\section{References}

[1] Sabbagh, C., Dumont, F., Robert, B., Badaoui, R., Verhaeghe, P. and Regimbeau, J.-M. (2011) Peritonea Volume Is Predictive of Tension-Free Fascia Closure of 
Large Incisional Hernias with Loss of Domain: A Prospective Study. Hernia, 15, 559-565. https://doi.org/10.1007/s10029-011-0832-y

[2] Balaphas, A., et al. (2016) Giant Hernias with Loss of Citizenship. Revue Médicale Suisse, 12, 1170-1173.

[3] Ventral Hernia Working Group, et al. (2010) Incisional Ventral Hernias: Review of Literature and Recommendations Regarding the Grading and Technique of Repair. Surgery, 148, 544-558. https://doi.org/10.1016/j.surg.2010.01.008

[4] Trivellini, G., et al. (1984) Surgical Treatment of Large Eventrations. Study of a Technic Adapted to Disorders of Respiratory Compliance. Chirurgie, 110, 116-122.

[5] Ramirez, O.M., Ruas, E. and Dellon, A.L. (1990) “Components Separation” Method for Closure of Abdominal-Wall Defects: An Anatomic and Clinical Study. Plastic and Reconstructive Surgery, 86, 519-526.

https://doi.org/10.1097/00006534-199009000-00023

[6] Alam, N.N., Narang, S.K., Pathak, S., Daniels, I.R. and Smart, N.J. (2016) Methods of Abdominal Wall Expansion for Repair of Incisional Herniae: A Systematic Review. Hernia, 20, 191-199. https://doi.org/10.1007/s10029-016-1463-0 